Design and Combinatorial Development of Shield-1 Peptide Mimetics Binding to Destabilized FKBP12

Madsen, Daniel; Jørgensen, Frederik P.; Palmer, Daniel; Roux, Milena E.; Olsen, Jakob V.; Bols, Mikael; Schoffelen, Sanne; Diness, Frederik; Meldal, Morten

Published in:

ACS Combinatorial Science

DOI:

10.1021/acscombsci.9b00197

Publication date:

2020

Document version

Publisher's PDF, also known as Version of record

Document license:

Other

Citation for published version (APA):

Madsen, D., Jørgensen, F. P., Palmer, D., Roux, M. E., Olsen, J. V., Bols, M., Schoffelen, S., Diness, F., \&

Meldal, M. (2020). Design and Combinatorial Development of Shield-1 Peptide Mimetics Binding to Destabilized FKBP12. ACS Combinatorial Science, 22(3), 156-164. https://doi.org/10.1021/acscombsci.9b00197 


\section{Design and Combinatorial Development of Shield-1 Peptide Mimetics Binding to Destabilized FKBP12}

Daniel Madsen, Frederik P. Jørgensen, Daniel Palmer, Milena E. Roux, Jakob V. Olsen, Mikael Bols, Sanne Schoffelen, Frederik Diness, ${ }^{*}$ and Morten Meldal*

Cite This: ACS Comb. Sci. 2020, 22, 156-164

Read Online

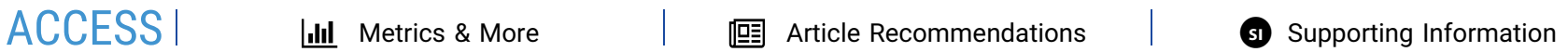

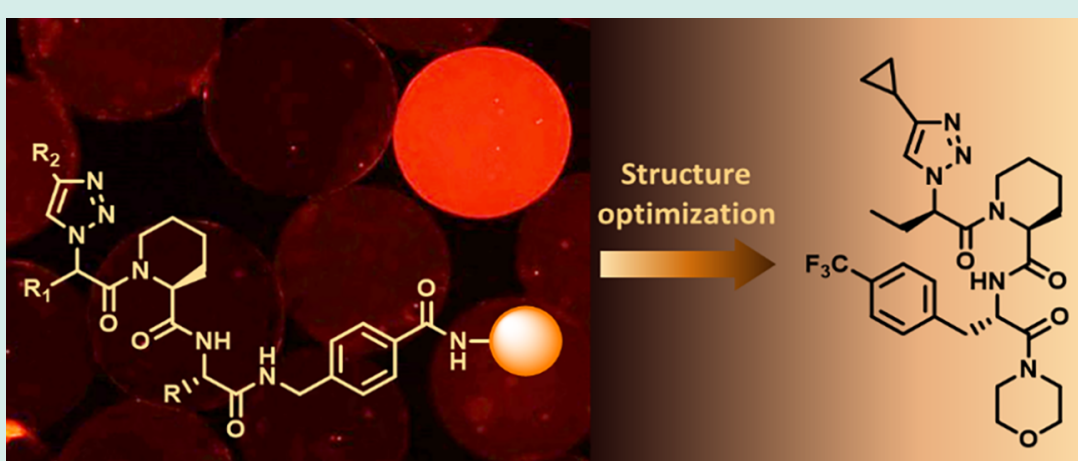

ABSTRACT: On the basis of computational design, a focused one-bead one-compound library has been prepared on microparticleencoded PEGA $\mathrm{A}_{1900}$ beads consisting of small tripeptides with a triazole-capped $\mathrm{N}$-terminal. The library was screened towards a double point-mutated version of the human FKBP12 protein, known as the destabilizing domain (DD). Inspired by the decoded library hits, unnatural peptide structures were screened in a novel on-bead assay, which was useful for a rapid structure evaluation prior to off-bead resynthesis. Subsequently, a series of 19 compounds were prepared and tested using a competitive fluorescence polarization assay, which led to the discovery of peptide ligands with low micromolar binding affinity towards the DD. The methodology represents a rapid approach for identification of a novel structure scaffold, where the screening and initial structure refinement was accomplished using small quantities of library building blocks.

KEYWORDS: destabilizing domain, one-bead one-compound library, encoded beads, solid-phase synthesis

\section{INTRODUCTION}

The engineered and double point-mutated version of the human FK506-binding protein, F36V-L106P-FKBP12, is known as the destabilizing domain (DD). ${ }^{1}$ This domain can confer its instability to proteins-of-interests (POIs) when fused to it. ${ }^{2,3}$ When expressed in cells, the DD and the fusion protein will rapidly be degraded by the proteasome, resulting in low levels of the POI. ${ }^{1}$ Upon stabilization of the DD by addition of a small ligand, the degradation is reduced, which induces a dose-dependent accumulation of the POI within the cell. Hereby, the level of gene product can be chemically controlled, creating a powerful tool for interrogating biological systems. The compound Shield-1 (Shld1, Figure 1) is a small molecule derived from the natural ligand FK506 and has been shown to interact selectively with the DD. ${ }^{4,5}$ The Shld1-DD system has proven its vast utility in several biological systems, including living mice, ${ }^{6}$ plants, ${ }^{7,8}$ parasites, ${ }^{9-11}$ mammalian cells, ${ }^{1}$ and yeast. ${ }^{12}$ Previous studies have reported $K_{\mathrm{i}}$ values of $7.5 \mathrm{nM}$ from in vitro experiments between Shld1 and the DD. ${ }^{13}$ However, in plant systems genetically modified to express DD-
POI fusion proteins, high concentrations of Shld1 were required to induce accumulation of the destabilized complex. ${ }^{7}$ In studies with rice and wheat, treatment with 3-10 $\mu \mathrm{M}$ Shld1 solutions was required to achieve sufficient diffusion of the small Shld1 molecule through the plant cuticle and subsequent stabilization of DD-POI. ${ }^{8}$ A recent study highlighted the challenges of improving the plant cell penetrating properties of Shld1 derivatives, while preserving sufficient potency of the modified compounds. ${ }^{13}$ In connection to this project, alternative and novel stabilizing compounds with a less demanding synthetic route were envisioned. We describe, in this study, a method for using computational design, combined with a series of encoded combinatorial synthesis and screening

Received: November 21, 2019

Revised: January 30, 2020

Published: February 6, 2020 


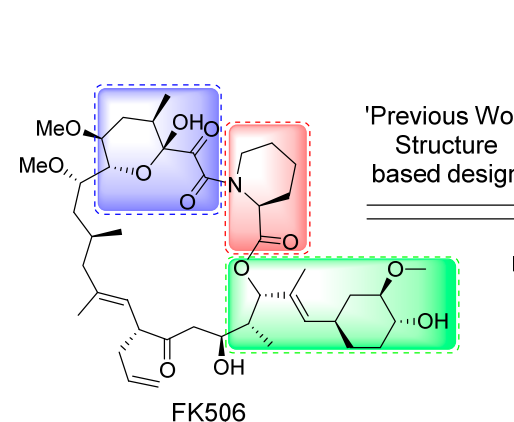

Ligand for FKBP12

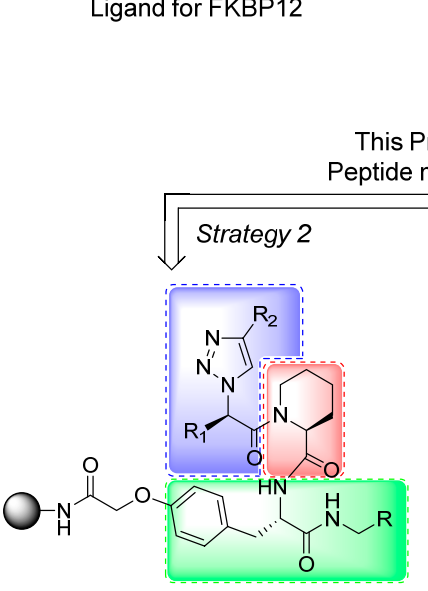

2
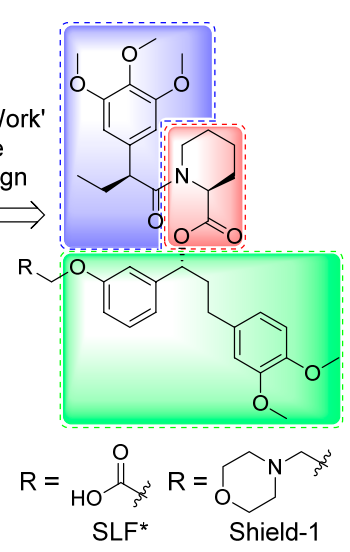
Ligands for DD

\section{ics} Strategy 1

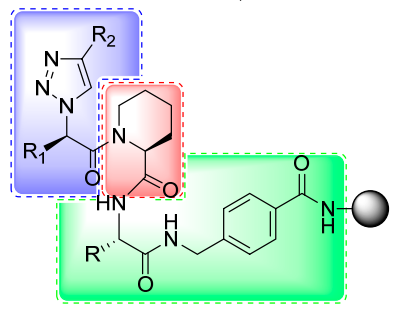

1
Figure 1. Illustration of the development process for small molecule peptide mimetics and their respective attachment strategies. Prior to this study, DD-ligands, such as SLF* and Shield-1 (Shld1), were derived by structure-based design originating from the natural product FK506. Strategy 1 of the peptide mimetics involve standard solidphase synthesis in the $C$ - to $N$-direction, whereas in strategy 2, the ligand is anchored via a carboxylate linker attached to a tyrosine sidechain.

steps, as a rapid approach for developing new peptide-based ligands that stabilize the DD protein.

The FKBPs have been described as being "undruggable" for low molecular mass ligands with sufficient affinity, ${ }^{14}$ and most synthetic ligands of the FKBPs are derived from the natural product FK506 (Figure 1). Reported strategies include the use of macrocyclic peptide scaffolds ${ }^{15,16}$ and structure-based design of the FK506-FKBP12 pharmacophore. ${ }^{17}$ Different synthetic approaches have been reported for the synthesis of Shld1-like structures, in which chiral reducing agents, ${ }^{18}$ chiral auxiliaries, $^{19}$ the addition of stoichiometric amount of chiral complexing agents, ${ }^{20}$ and chromatographic separation of diastereomers ${ }^{5}$ have been utilized to obtain the enantiopure compounds. Inspired by the structural insight of these previous studies, ${ }^{1,5}$ we selected an in silico method for the design of new scaffolds that mimic the binding of Shld1 to the DD. The scaffolds should be assembled from simple amino acid building blocks by solid-phase peptide synthesis. The resulting initial structural design (vide infra) is presented in Figure 1 and consists of tripeptides capped at the $\mathrm{N}$-terminal with a triazole by CuAAC click chemistry.

We implemented and report here on a new on-bead binding assay using templated prefolding of the labeled, disordered protein target for the evaluation of the target binding by the new scaffolds. The assay allowed rapid scaffold optimization. The solid-supported ligands could be synthesized in parallel using only $10 \mathrm{mg}$ of resin per compound, thus enabling a rapid hit structure elucidation prior to off-bead resynthesis. The scaffold allowed synthesis of one-bead-one-compound (OBOC) libraries and comprised two strategies with different points of attachment to the support (1 and 2, Figure 1). The compound $\mathrm{SLF}^{* 5}$ (Figure 1), which is closely related to Shld1, has a carboxylic acid handle for attachment to the solid support and was selected as a reference for studying the on-bead target interaction.

The screening of a focused combinatorial library, synthesized on optically encoded beads containing fluorescent microparticles, ${ }^{21}$ facilitated rapid identification of hit compounds through simple optical decoding. This library encoding approach is suitable for synthesis and screening of small- to medium-sized libraries (500-50 000 beads) and permits, as do other bead-based encoding technologies, ${ }^{22-25}$ the identification of library members binding to the fluorescent target. The method relies on the fact that each bead in the library carries a unique distribution of microparticles as a code obtained by recording the microparticle coordinates that distinguish it from all other beads in the library. The encoded library technology is particularly useful for bead-based libraries of compounds for which conventional mass-spectrometry methods are complicated and inefficient, as was the case in the presented study. ${ }^{26}$

The structural insight gained from the on-bead assay assisted the design of 19 unnatural peptides which were screened in a competitive fluorescence polarization assay, and our findings has led to the discovery of novel and small unnatural peptides with low micromolar binding affinity towards the DD.

\section{RESULTS AND DISCUSSION}

With the aim of developing a core structure with modifiable moieties for combinatorial library development, a tripeptide with a triazole capped $\mathrm{N}$-terminal was designed by computer modeling to adopt a similar binding pose to that of Shld 1 in the DD binding site. ${ }^{5}$ The reference compound $\mathrm{SLF}^{*}$ and an example of the computational designed ligand $\mathbf{1}$ is depicted in Figure 2. Modeling was performed with Molecular Operating

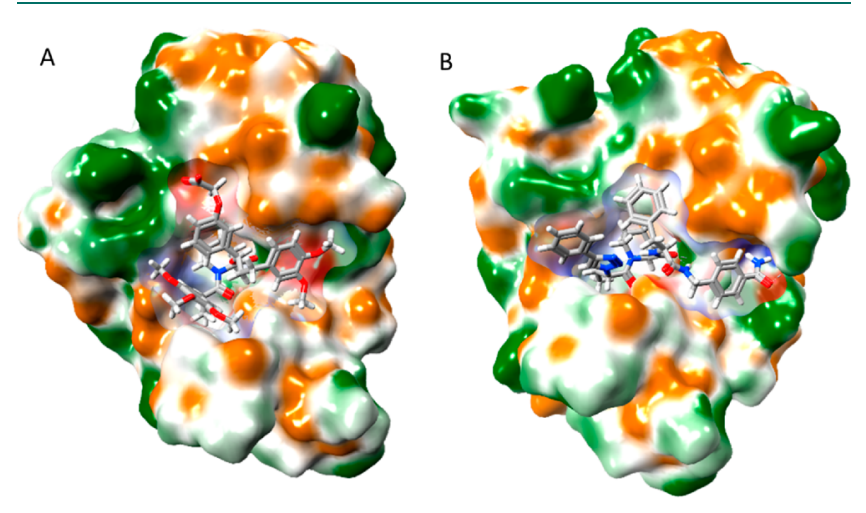

Figure 2. Comparison of the binding modes of $\operatorname{SLF}^{*}$ (A) and an example of peptide mimetic 1 (B) to the crystal structure of the SLF*stabilized F36V-FKBP12 complex. In the modeling of the complex between F36V-FKBP12 and the peptide mimetic, key features of the ligand were maintained while building the structure. The watersoaked complexes of F36V-FKBP12 with the peptide mimetic was subjected to several rounds simulated annealing (MOE, Amber 10, $\mathrm{ETH}$; $T=430 \mathrm{~K}, 1 \mathrm{~ns}, 430-230 \mathrm{~K}, 1 \mathrm{~ns}, 230 \mathrm{~K}, 3 \mathrm{~ns}$ ). In the first round, the coordinates of F36V-FKBP12 were fixed, and in the subsequent rounds, the contact residues in F36V-FKBP12 were relaxed to allow an induced fit of F36V-FKBP12 and the ligand. 
Scheme $1^{a}$

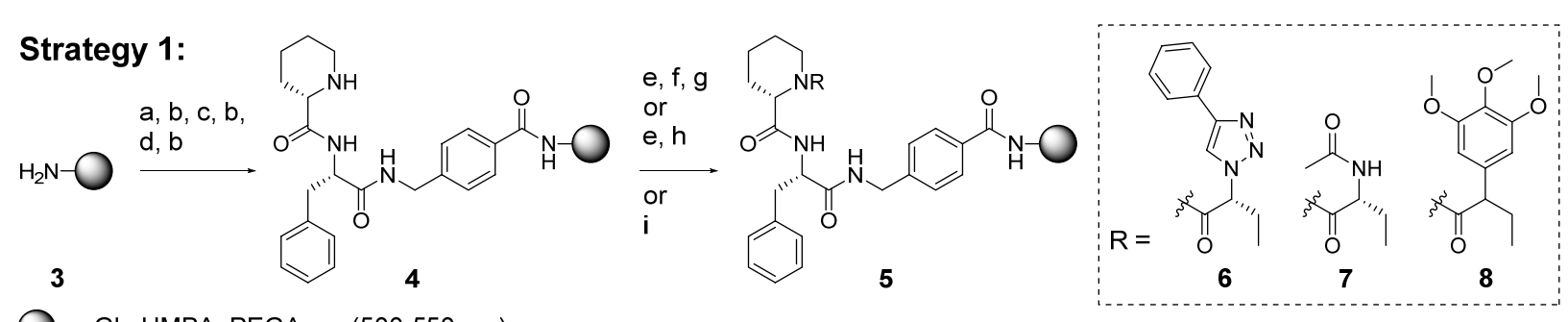

$=$ Gly-HMBA, PEGA $1900(500-550 \mu \mathrm{m})$

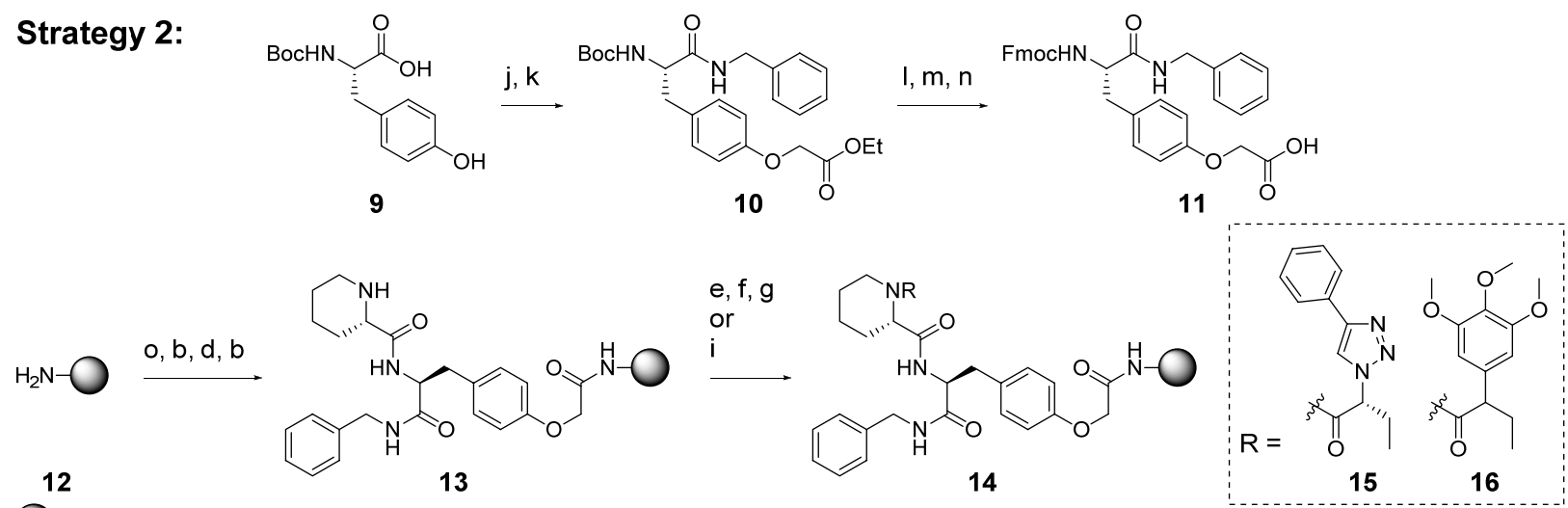

$\mathrm{O}=$ Gly-HMBA, PEGA $1900(500-550 \mu \mathrm{m})$

Reference:

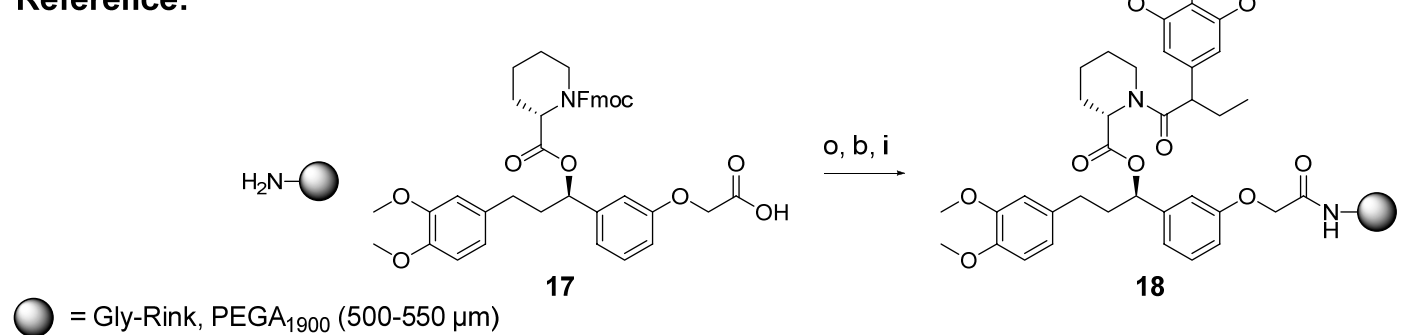

${ }^{a}$ Reagents and conditions: (a) Fmoc-4-Amb-OH, TBTU, NEM, DMF, 2 h; (b) piperidine/DMF (1:4), 20 min; (c) Fmoc-L-Phe-OH, TBTU, NEM, DMF, 2 h; (d) Fmoc-L-Pip-OH, TBTU, NEM, DMF, 2 h; (e) Fmoc-D-Abu-OH, TBTU, NEM, DMF, 2 h, then piperidine/DMF (1:4), 20 min; (f) imidazole-1-sulfonyl azide hydrochloride, $\mathrm{CuSO}_{4}, \mathrm{~K}_{2} \mathrm{CO}_{3}, \mathrm{H}_{2} \mathrm{O}, 16 \mathrm{~h}$; (g) phenylacetylene, $\mathrm{CuSO}_{4}$, ascorbic acid, $\mathrm{t}-\mathrm{BuOH} / \mathrm{H}_{2} \mathrm{O}(3: 1), 16$ h; (h) $\mathrm{Ac}_{2} \mathrm{O} /$ pyridine/DMF (1:1:8), $30 \mathrm{~min}$; (i) 2-(3,4,5-trimethoxyphenyl)butanoic acid, TBTU, NEM, DMF, 2 h; (j) benzylamine, PyBOP, NEM, DMF, 1 h, 99\%; (k) ethyl-2-bromoacetate, $\mathrm{Cs}_{2} \mathrm{CO}_{3}$, DMF, $3 \mathrm{~h}, 86 \%$; (l) TFA $/ \mathrm{CH}_{2} \mathrm{Cl}_{2}(1: 1), 0{ }^{\circ} \mathrm{C}$ to rt, $60 \mathrm{~min} ;(\mathrm{m}) \mathrm{Fmoc}-\mathrm{Cl}, \mathrm{Na}{ }_{2} \mathrm{CO}_{3}$, dioxane $/ \mathrm{H}_{2} \mathrm{O}(2: 1), 0{ }^{\circ} \mathrm{C}$ to rt, $1.5 \mathrm{~h}, 93 \%$ (2 steps); (n) $\mathrm{MgI}_{2}$, THF, mw $120{ }^{\circ} \mathrm{C}, 15 \mathrm{~min}$, quant; (o) TBTU, NEM, DMF, $2 \mathrm{~h}$.

Environment (MOE, CCG) using the crystal structure of SLF* bound to F36V-FKBP12 (PDB 1BL4) as a starting point. ${ }^{5}$ Upon fixing the coordinates of the protein, the SLF* compound was replaced with peptide mimetics, soaked in a water droplet, and relaxed by energy minimization as described in detail in the Supporting Information. Modifications were explored on key features of the peptides during structural development. The pipecolic moiety is located in a groove on the active site of the protein and has in several studies been shown to be important for binding to the protein. $8,14,17,18,27$

A range of alternative ring sizes and substitution patterns were sampled during the modeling, but the pipecolic residue presented a superior fit to the pocket. Originating from an $\alpha$ aminobutyric acid, the ethyl part adjacent to the triazole is designed to dock into the hydrophobic cavity formed after the F36V mutation, ${ }^{5}$ and various lengths of alkyl groups were investigated and selected to explore its capacity. Those alkyl groups providing favorable interactions in the pocket during modeling were included in subsequent library synthesis. For the remaining residues, interaction over the entire binding site was evaluated and structural components displaying appropriate contact with the protein were selected for combinatorial library synthesis.

The design of the peptide mimetic scaffold allowed usage of the large pool of natural and unnatural amino acid building blocks in combination with the orthogonal CuAAC click chemistry to survey the pharmacophore interactions. To screen for optimal interaction with the protein for the immobilized substrates in the library, two different linker strategies were employed. As presented in Scheme 1, strategy 1 involve standard solid-phase peptide synthesis (SPPS) in the $C$ - to $N$ direction. Whereas, strategy 2 is anchored via a linker piece from the tyrosine side chain, protruding from the other end of the compound and resembling the part of the $\mathrm{SLF}^{*}$ used for 
immobilization of 18. Presumably, attachment from this part of the peptide would exhibit a smaller degree of steric interaction with the DD. To initially verify the scaffold design and test the two linking strategies, 2-3 examples of the scaffold linked by each of the methods were synthesized on solid support (Scheme 1). The building block for the strategy 2 linker was synthesized by coupling of 9 with benzylamine, followed by a substitution of ethyl 2-bromoacetate to give 10. A Boc to Fmoc protection group exchange of $\mathbf{1 0}$ was performed, and after ester hydrolysis orthogonal to the Fmoc protection group, ${ }^{28} 11$ was formed in five steps with an overall yield of $79 \%$, starting from Boc-protected L-tyrosine. A set of solid-supported compounds were then synthesized on a PEGA $_{1900}$ resin $(500-550 \mu \mathrm{m})^{29}$ and subjected to a maltose binding protein (MBP) fused DD, which had been fluorescently labeled with the rhodamine-X fluorophore (MBP-DD-(ROX)). The MBP was fused to the $\mathrm{DD}$ to prevent aggregation of the destabilized protein and to increase the overall polarity of the protein, hence reducing the nonspecific binding. The unique swelling properties of the porous $\mathrm{PEGA}_{1900}$ resin in aqueous media enabled the large fusion protein to gain access to the bead interior. ${ }^{30}$ As a reference compound for the initial test screen, a solidsupported version of the SLF* compound was synthesized by adapting known procedures (see Supporting Information). ${ }^{18}$

The carboxylic acid part of the SLF* has been shown to have minor influence on the binding to the DD protein and has previously been used as an anchor for solid-support while retaining its interaction with the protein. ${ }^{31}$ To estimate any level of nonspecific binding, three different control tripeptides presenting negative, positive, and neutral charge (19-21, see Figure 3) were tested in the on-bead binding assay. As

\section{On-Bead Screen}

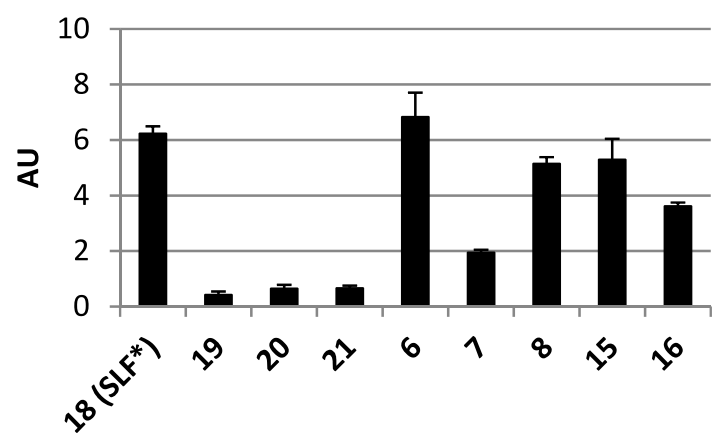

Figure 3. Single concentration binding tests of solid-supported ligands subjected to a fluorescently labeled DD protein $(550 \mathrm{nM})$ for $16 \mathrm{~h}$. The three tripeptides 19 (Ac-Glu-Phe-Gly-OH), 20 (Ac-LysPhe-Gly-OH), and 21 (Ac-Ala-Phe-Gly-OH) were attached to the PEGA resin via the HMBA linker and used as controls for nonspecific binding.

presented in Figure 3, compounds from both attachment strategies containing the 2-trimethoxyphenylbutyric acid building block showed significant binding, albeit with lower affinity than the reference solid-supported $\operatorname{SLF}^{*}(\mathbf{1 8})$ in the single concentration on-bead screen. The acetylated compound 7 bound poorly to the protein, while the derivatives 6 and 15 , containing a phenyltriazole, presented good binding, as measured by the fluorescence due to the resin-bound MBPDD-(ROX). With a relatively high effective ligand concentration of approximately $3.4 \mathrm{mM}$, a situation of on-bead binding saturation is presumably observed for strong binding ligands. Indeed, compound $\mathbf{6}$ provided the same if not more fluorescence intensity than resin bound SLF*. However, these results indicated that the triazole-based mimetics of the 2trimethoxyphenylbutyric acid would be a good starting point for development of a combinatorial library.

The main advantage of this approach was that the azido acid could be generated on bead from amino acid building blocks, hereby removing the need for introducing a stereocenter through enantioselective alkylation of a range of phenylacetic acid derivatives. ${ }^{19,20}$ Because of the anticipation of slightly better interaction and the ease of synthesis, strategy 1 was selected for the development of the combinatorial library.

Library Screening. Using the split-and-mix approach, a library of in total 896 different compounds were synthesized on PEGA $_{1900}$ beads $(500-550 \mu \mathrm{m})$ that were optically encoded by MicroParticleMatrix (MPM)-encoding (Figure 4). ${ }^{32}$ Encoding of the immobilized peptides was performed by

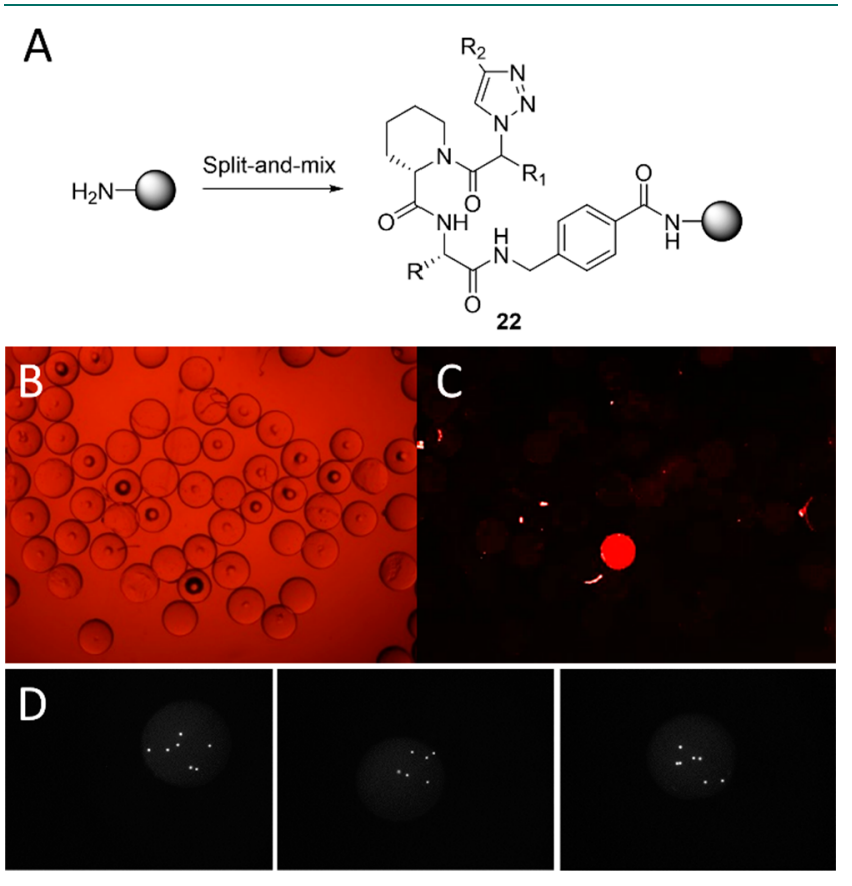

Figure 4. Development and screening of encoded one-bead onecompound library. (A) Using the split-and-mix approach, an 896member library was synthesized on encoded PEGA 1900 beads (500$550 \mu \mathrm{m}$ ) using the same conditions as presented in Scheme 1. (B and C) Bright field and fluorescence images, respectively, of library beads after being subjected to a $1 \mu \mathrm{M}$ solution of Shld1-stabilized MBP-DD(ROX) for $48 \mathrm{~h}$. The images presents a clear hit bead among inactive beads. (D) Typical encoded bead used in the present study. Three orthogonal images of the same bead are recorded and used in construction of the 3-dimensional matrix represented by the microparticle coordinates. These are used as a unique digital code to identify the structure of the compound immobilized on the bead. The code of the bead is recorded at each synthetic step and used for hit identification.

recording three orthogonal images of every bead in each portion of the split process, to generate a microparticle coordinate matrix used as a barcode for the individual beads. The images were recorded using a custom-made MPM decoding instrument comprising a solid state laser, three CCD-cameras with telecentric optics and a carousel for individual bead handling. ${ }^{21}$ Subsequently, the isolated hit- 
beads were recorded and the 3-dimensional matrix represented by their microparticle coordinates were determined, allowing tracking through the synthetic history of the immobilized compound. ${ }^{21}$ Pipecolic acid and 4-(aminomethyl) benzoic acid were maintained as structural features during the library development. The remaining amino acid building blocks were varied and coupled using either coupling reagents TBTU or HATU. Subsequently, a solid-phase diazotransfer to the $\mathrm{N}$ terminal amine was carried out to form the corresponding azide, ${ }^{33}$ which in turn was reacted by the CuAAC click reaction using various alkynes (see Table S1 for the full list of library building blocks).

The selection of structure variations of $R, R_{1}$, and $R_{2}$ were guided by computational design as described above. The moieties on the R-position were primarily aliphatic and aromatic cyclic structures, whereas the $\mathrm{R}_{1}$-position consisted of small linear and branched alkyl groups. Presumably, the enlargement of the hydrophobic cavity formed upon F36V mutation of FKBP12 could allow binding of longer alkyl groups, particularly in combination with the less bulky triazole moiety, as compared to the reference trimethoxy phenylbutyric acid residue of SLF*. To induce the correct folding of the protein, the MBP-DD-(ROX) was preincubated for $30 \mathrm{~min}$ with a stoichiometric amount of Shld1 prior to the incubation of the library beads. A final concentration of $1 \mu \mathrm{M}$ MBP-DD(ROX) was used, and the beads were incubated for $48 \mathrm{~h}$ to establish equilibrium between the individual compounds in a binding-competition assay. As presented in Figure 4, a clear difference in interaction with the protein among the solidsupported ligands was observed, which was visualized by recording the fluorescence intensity of the bead collections. The binding was quite selective and provided a number of bright beads on a background of many inactive beads. The identified hit-beads were manually sorted based on their apparent fluorescence intensity. The structures attached to the isolated encoded hit-beads were decoded giving a total of 16 different hit-structures out of 17 isolated beads (see Supporting Information for all the hit structures). ${ }^{21}$ Nine of the hit compounds contained a 4-trifluoromethyl phenylalanine derivative as the second amino acid. In addition, 12 compounds had an ethyl group on the $\mathrm{R}_{1}$-position and primarily either a 4-methoxy phenyl or a cyclopropyl on the $\mathrm{R}_{2}$-position.

On-Bead Assay and Structure Evaluation. To distinguish and verify the potency among the structures of the solidsupported hit compounds, a well-based on-bead binding assay was employed. ${ }^{34}$ Guided by the results from the library screen, a set of immobilized substrates were resynthesized on PEGA $_{1900}$ beads $(125-200 \mu \mathrm{m})$. The beads were singly sorted into a 96-well plate using a COMPAS bead sorter (Union Biometrica) and subjected to a dilution series of MBP-DD(ROX). After $16 \mathrm{~h}$ of incubation, the fluorescence intensity was measured on wide-field fluorescence microscope. The individual binding profile of the resynthesized peptide mimetics was compared to the solid-supported SLF* and graded in a scale from 1 to 4 (Table 1). In general, it was found that the most abundant substituents from the hits in the library screen, also proved to be the most potent in the bead assays. Compounds containing a 4-trifluoromethyl phenylalanine as the second amino acid, and an ethyl group on the $\mathrm{R}_{1}$-position displayed the most promising binding profile. On the $R_{2}$ position, neither the phenyl group of $\mathbf{2 3}$ of nor the 3-benzoic acid of $\mathbf{2 4}$ were identified as hits during the screening but were
Table 1. On-Bead Screening of Compounds Derived from the Hits Identified through Optically Encoded Split-andMix Library Technology

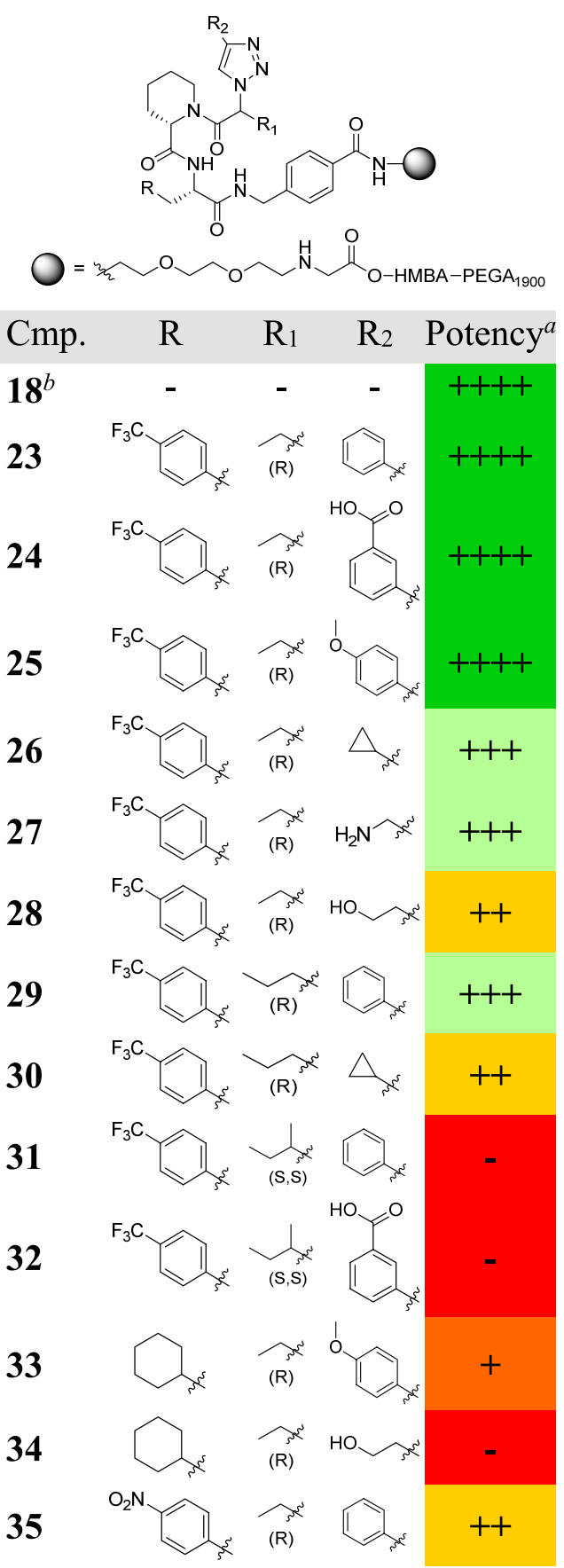

${ }^{a}$ Binding profile of the peptide mimetics was compared to the solidsupported SLF* and their potency was graded in a scale from 1 to 4 . ${ }^{b}$ Immobilized SLF* (see Scheme 1).

selected for comparison with the binding of $\mathbf{2 5}$. These two compounds displayed a comparable binding profile to the anisole of 25, which indicated that aromatic moieties on this position was preferred. Resynthesis of the peptide mimetics in the solid-supported screening format was realized on a low micromolar scale, requiring a minimal amount of the unnatural amino acid building-blocks, which in combination with the library screening gave an efficient and rapid structure evaluation. 
Table 2. $K_{\mathrm{i}}$ Values of Resynthesized Peptide Mimetics 36-54

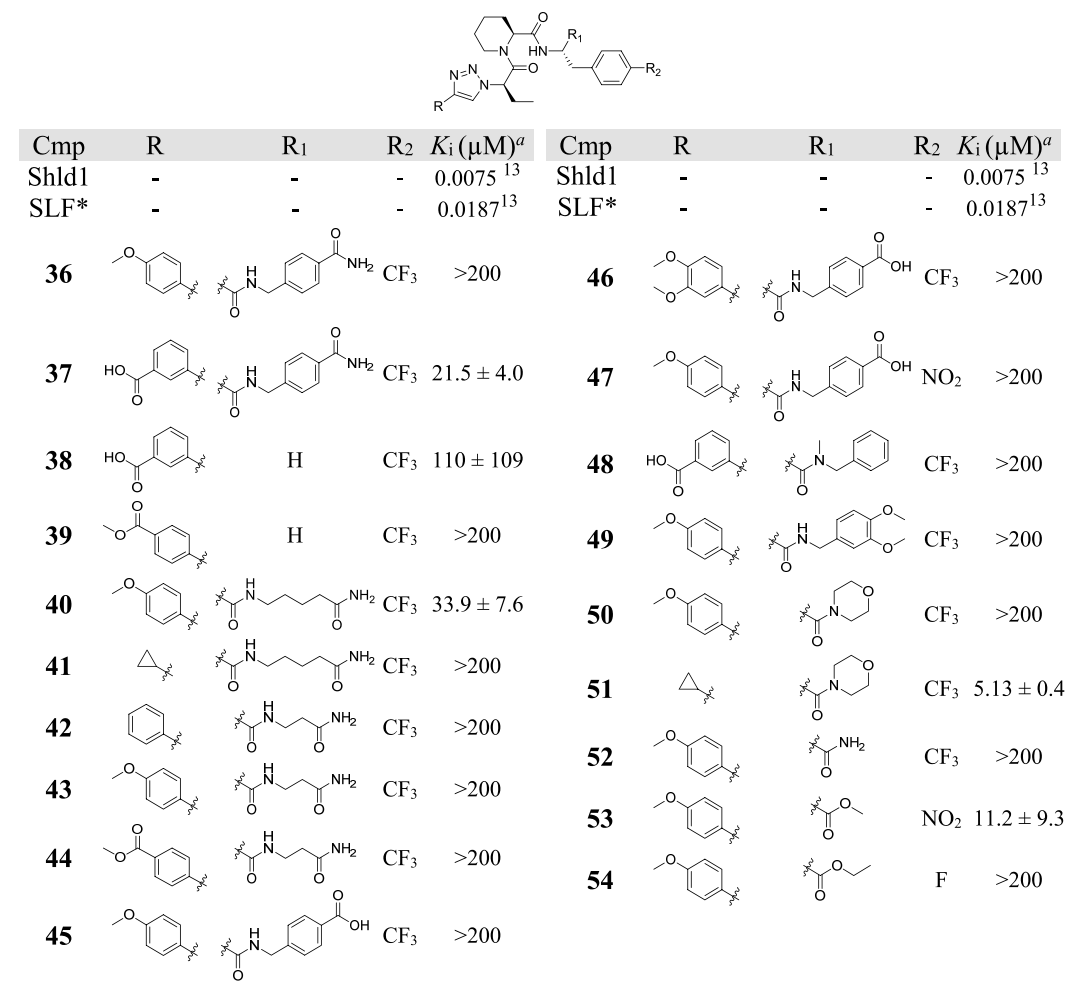

${ }^{a} \mathrm{~A}$ competitive fluorescence polarization assay was used to obtain the reported $K_{\mathrm{i}}$ values \pm SD from three independent experiments.

Off-Bead Peptide Resynthesis and Screening. The next step was to survey the nonlinked scaffold for binding to the MBP-DD in solution. On the basis of the results obtained from the well-based on-bead assay, a set of 19 unnatural peptides were synthesized in solution or from solid-support (see Supporting Information). Using the same synthetic approach as presented in Scheme 1, the compounds containing a primary amide or a benzoic acid on the $C$-terminal of the peptides were synthesized from the Rink-amide linker and the 2-chloro trityl linker, respectively. The solid-supported compounds were cleaved upon acid treatment to yield the desired peptide mimetics in modest to excellent yields. The compounds with no handle for solid support were synthesized in 3-4 steps in solution. For this, primary and secondary amines were coupled with a substituted Boc-protected Lphenylalanine derivative. Subsequently, the Boc-group was removed under acid treatment and the Boc-pipecolic acid was coupled using HBTU. In a subsequent one-pot protecting group removal and coupling procedure, an azido modified $\mathrm{D}-\alpha$ aminobutyric acid was introduced, and the final triazole was formed in a CuAAC reaction. Variation on the $\mathrm{R}_{1}$-position (Table 2) included moieties of different size, polarity, and charge. The 19 peptide mimetics were screened towards the MBP-DD using a recently described fluorescence polarization competition assay. ${ }^{13}$ Directed by the results from the on-bead assay, the unbound versions of $\mathbf{2 4}$ and $\mathbf{2 5}$ were synthesized and tested. The most promising of these compounds was the aminomethyl benzamide 37 , which showed a $K_{\mathrm{i}}$ of $21.5 \mu \mathrm{M}$. It was found that omitting a $\mathrm{R}_{1}$-substituent (38) reduced the activity 5 -fold, whereas enhancing the lipophilicity by changing the carboxylic acid to an ester or omitting the amide on the $\mathrm{R}_{1}$ substituent led to loss of activity. Many of the resynthesized ligands showed poor solubility under the conditions of the assay, which is a crucial property for biological activity. This influenced the outcome of the fluorescence polarization competition assay and complicated the accurate assessment binding constants. Reducing the lipophilicity of 36 revealed that changing to an aliphatic amide substituent (40), some activity could be achieved and that this was lost when switching to a lipophilic substituent (49). However, the effect appeared not only to be enhanced solubility, since benzoic acid derivatives $(45-47)$ or shorter aliphatic amide substituent (42-44) in the $R_{1}$-position only led to inactive derivatives. Compound 40 exhibited a $K_{\mathrm{i}}$ of $33.9 \mu \mathrm{M}$ and has a primary amide positioned in a similar distance from the pipecolic core as compound 37, suggesting that a hydrogen bond donor in this position may be advantageous to the binding of the protein. Testing rather small moieties on the $R_{1}$-position in combination with or without changing the $\mathrm{R}-$ and $\mathrm{R}_{2}$ substituents (50-54) interestingly revealed the two most potent compounds in the series ( 51 and 53) with $K_{\mathrm{i}}$-values of 5.13 and $11.2 \mu \mathrm{M}$, respectively. Both compounds are among the most hydrophilic, but again, this effect is not the only determining factor, as introducing the aliphatic amide substituent found the active derivative $\mathbf{4 0}$ in the $\mathrm{R}_{1}$-position of 51 led to loss of activity (41). This underlines that further optimization of the scaffold has to be multidimensional, which exactly is the strength of combinatorial screening. However, the presented data demonstrates that the combination of computational design and combinatorial synthesis and screening is a powerful concept for developing new active scaffolds towards a given biological target.

Binding Evaluation of Peptide Ligands. The mode of interaction of the two ligands, 37 and $\mathbf{5 1}$ was analyzed in detail. The interactions between ligand 37 and F36V-FKBP12 involve two hydrophobic pockets (see Figure 5A). One for the 

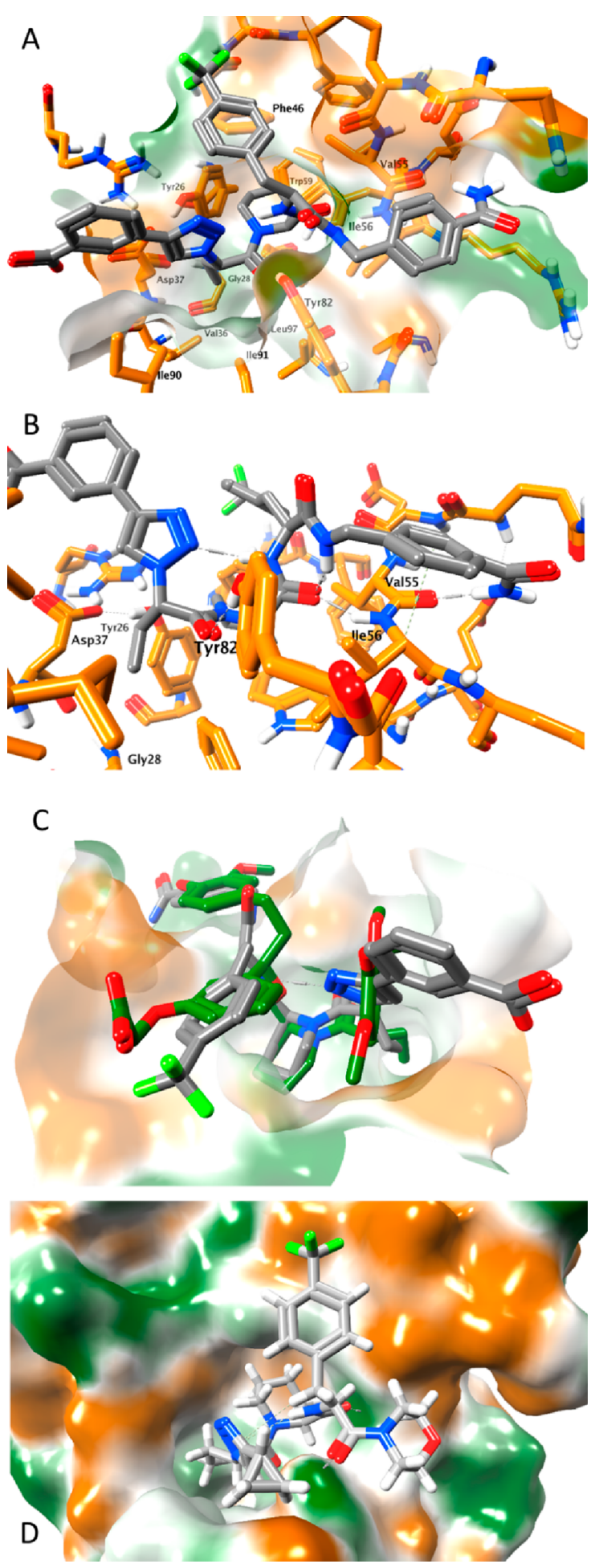

Figure 5. (A-C) Binding modes of compounds 37 and 51 (D) to the crystal structure of F36V-FKBP12 in the SLF*-stabilized complex presented by computational modeling. (A) Contact residues of interaction between 37 with F36V-FKBP12. (B) Hydrogen bond network of interacting residues between the complex 37 and F36VFKBP12. (C) The overlay of SLF* of the crystal structure (green) and ligand 37 (gray) in the binding pocket of F36V-FKBP12. (D) The alternate binding mode presented by compound $\mathbf{5 1}$.

ethyl group, which interact with Ile-91, Gly-28, Val-36, Phe-99, Tyr-26, and Leu-97 of F36V-FKBP12, and one for the pipercolic acid, which is lined by Phe-46, Phe-99, Tyr-26, Ile-
56, and Val-55, with Trp-59 positioned at the bottom of the pocket. In addition, the 4-trifluoromethyl-L-phenylalanine interact with Phe-46, and the aromatic ring of the amidomethylbenzamide residue interact with Ile-56. The bound 37 show a set of crucial hydrogen bonds. The $C$ terminal amide binds to the carbonyl group of Val-55 while the $\mathrm{NH}$ of the amidomethylbenzamide binds internally to the carbonyl of the pipecolic amide. This in turn binds amide $\mathrm{NH}$ of Ile-56. Tyr-82 forms a hydrogen bond to the deeply buried carbonyl of the triazole modified butyric acid, while the triazole forms a hydrogen bond to the $\mathrm{NH}$ of the $\mathrm{Phe}\left(\mathrm{CF}_{3}\right)$ residue. Overall the fit of 37 is not as tight as that of SLF*. ${ }^{5,13}$ The optimized interaction of the three peripheral aromatic rings seem to lift the core pipecolic amide and $\alpha$-aminobutyric acid residue slightly from their binding pockets, since it cannot reach over the rim of the binding pocket. Hereby, interaction with the protein is compromised, and as visualized in Figure $5 \mathrm{C}$, the SLF* is in significant closer contact with the protein in the deep hydrophobic binding pocket. The complex of F36VFKBP12 with compound $\mathbf{5 1}$ presents an alternative binding mode in which the cyclopropane ring interacts with Ile-90, His97, and Tyr-82 and the triazole binds perpendicularly to the $\mathrm{NH}$ of the Phe $\left(\mathrm{CF}_{3}\right)$. This redirects Tyr- 82 to form a hydrogen bond to the carbonyl of $\mathrm{Phe}\left(\mathrm{CF}_{3}\right)$, allowing optimal interaction of the morpholine residue with Ile-56. Surprisingly, the smaller compound $\mathbf{5 1}$ showed the highest binding affinity. However, since all peripheral residues of $\mathbf{5 1}$ are relatively small, they may allow optimal interaction of the core pipecolic amide and $\alpha$-aminobutyric acid residues with the F36V-FKBP12. These observations suggest that the scaffold could be even further improved by extending the core framework to position the peripheral aromatic residues at more favorable regions of the interaction interface, thus providing a better match of binding to the active site of the DD.

In summary, an 896-member one-bead one-compound library was synthesized on microparticle encoded PEGA 1900 beads using the split-and-mix approach. The library was screened towards a fluorescently labeled version of the DDprotein and the screen resulted in 16 different structures, which were identified as hits and deconvoluted using in-house developed decoder equipment. General trends among the hits included the presence of a 4-trifluoromethyl-L-phenylalanine as the second amino acid and an ethyl-group on the $\mathrm{R}_{1}$-position (Figure 4) originating from an $\alpha$-D-aminobutyric acid. Guided by the library hits, solid supported peptide mimetics were resynthesized on small scale and screened in a novel on-bead binding format to confirm and distinguish the potency among the library hits. The structure information was then used for the synthesis of 19 peptide mimetics. By exploiting the fixed stereochemistry of the unnatural amino acids, the small peptide-like compounds were synthesized in few steps using either solution- or solid-phase synthesis. The peptide mimetics were tested in a competition fluorescence polarization assay and one compound with a low micromolar binding affinity towards the DD was identified. Analysis of the identified low potency ligand (37) indicates that binding may be partly compromised by the small displacement of the pipecolic and butyric acids caused by rim interaction of the peripheral residues. This may also add to the explanation, why a small compound like 51 bind better than 37, while displaying less interaction. Our high-throughput screening approach to identify a novel peptide-like structure scaffold for the DD has involved the combination of computational modeling, 
combinatorial library screening, and on-bead structure evaluation.

With a focus on improving the solubility of the peptide mimetics, the identified ligand $\mathbf{5 1}$ could serve as a starting point for development of unnatural peptides that mimic the Shld1-DD interaction. We envision that the present methodology can be applied to the synthesis and screening of larger peptide and nonpeptide libraries, where the screening and initial structure refinement can be accomplished routinely and rapidly using small quantities of library building blocks in a high throughput manner using the MPM-encoding technology.

\section{ASSOCIATED CONTENT}

\section{SI Supporting Information}

The Supporting Information is available free of charge at https://pubs.acs.org/doi/10.1021/acscombsci.9b00197.

Full description of the materials and methods, including HPLC, NMR, and MS data (PDF)

\section{AUTHOR INFORMATION}

\section{Corresponding Authors}

Frederik Diness - Department of Chemistry and Center for Evolutionary Chemical Biology, University of Copenhagen, 2100 Copenhagen, Denmark; ㅇo orcid.org/0000-0001-5098-7198; Email: fdi@chem.ku.dk

Morten Meldal - Department of Chemistry and Center for Evolutionary Chemical Biology, University of Copenhagen, 2100 Copenhagen, Denmark; 이이이.org/0000-0001-6114-9018; Email: meldal@chem.ku.dk

\section{Authors}

Daniel Madsen - Department of Chemistry and Center for Evolutionary Chemical Biology, University of Copenhagen, 2100 Copenhagen, Denmark

Frederik P. Jørgensen - Department of Chemistry, University of Copenhagen, 2100 Copenhagen, Denmark

Daniel Palmer - Department of Chemistry and Center for Evolutionary Chemical Biology, University of Copenhagen, 2100 Copenhagen, Denmark; ㅇ orcid.org/0000-0002-7954-9332

Milena E. Roux - Department of Biology, University of Copenhagen, 2100 Copenhagen, Denmark

Jakob V. Olsen - Department of Biology, University of Copenhagen, 2100 Copenhagen, Denmark

Mikael Bols - Department of Chemistry, University of Copenhagen, 2100 Copenhagen, Denmark; (1) orcid.org/00000002-6561-6952

Sanne Schoffelen - Department of Chemistry and Center for Evolutionary Chemical Biology, University of Copenhagen, 2100 Copenhagen, Denmark; 이이.org/0000-0003-2664-8561

Complete contact information is available at:

https://pubs.acs.org/10.1021/acscombsci.9b00197

\section{Author Contributions}

The manuscript was written through contributions of all authors. All authors have given approval to the final version of the manuscript.

Notes

The authors declare no competing financial interest.

\section{ACKNOWLEDGMENTS}

The University of Copenhagen has supported the present research through the UCPH-lighthouse program (CECB-
Lighthouse Grant 2013) and the free Research Council of Denmark (DFF-4184-00019).

\section{ABBREVIATIONS}

Ac, acetyl; Abu, $\alpha$-aminobutyric acid; Amb, aminomethylbenzoic acid; Boc, tert-butoxycarbonyl; Comp, compound; DD, destabilizing domain; DMF, N,N-dimethylformamide; FKBP, FK506-binding protein; Fmoc, 9-fluorenylmethyloxycarbonyl; HATU, 1-[bis(dimethylamino)methylene]-1N-1,2,3-triazolo[4,5-b]pyridinium 3-oxide hexafluorophosphate; HMBA, hydroxymethylbenzoic acid; MBP, maltose-binding protein; NEM, N-ethylmorpholine; NSB, nonspecific binding; PEGA, polyethylene glycol dimethyl acrylamide; Pip, pipecolic acid; POI, protein of interest; PyBOP, benzotriazole-1-yl-oxytripyrrolidinophosphonium hexafluorophosphate; ROX, 5(6)rhodamine-X; rt, room temperature; Shld1, Shield-1; SPPS, solid-phase peptide synthesis; SPS, solid-phase synthesis; TBTU, $N$-[(1H-benzotriazol-1-yl)(dimethylamino)methylene $]-N$-methyl methanaminium tetrafluoroborate; TFA, trifluoroacetic acid; THF, tetrahydrofuran

\section{REFERENCES}

(1) Banaszynski, L. A.; Chen, L.-c.; Maynard-Smith, L. A.; Ooi, A. G. L.; Wandless, T. J. A Rapid, Reversible, and Tunable Method to Regulate Protein Function in Living Cells Using Synthetic Small Molecules. Cell 2006, 126 (5), 995-1004.

(2) Chu, B. W.; Banaszynski, L. A.; Chen, L.-c.; Wandless, T. J. Recent Progress with FKBP-Derived Destabilizing Domains. Bioorg. Med. Chem. Lett. 2008, 18 (22), 5941-5944.

(3) Maynard-Smith, L. A.; Chen, L. C.; Banaszynski, L. A.; Ooi, A. G. L.; Wandless, T. J. A Directed Approach for Engineering Conditional Protein Stability Using Biologically Silent Small Molecules. J. Biol. Chem. 2007, 282 (34), 24866-24872.

(4) Grimley, J. S.; Chen, D. A.; Banaszynski, L. A.; Wandless, T. J. Synthesis and Analysis of Stabilizing Ligands for FKBP-Derived Destabilizing Domains. Bioorg. Med. Chem. Lett. 2008, 18 (2), 759761.

(5) Clackson, T.; Yang, W.; Rozamus, L. W.; Hatada, M.; Amara, J. F.; Rollins, C. T.; Stevenson, L. F.; Magari, S. R.; Wood, S. A.; Courage, N. L.; Lu, X.; Cerasoli, F.; Gilman, M.; Holt, D. A. Redesigning an FKBP-Ligand Interface to Generate Chemical Dimerizers with Novel Specificity. Proc. Natl. Acad. Sci. U. S. A. 1998, 95 (18), 10437-10442.

(6) Banaszynski, L. A.; Sellmyer, M. A.; Contag, C. H.; Wandless, T. J.; Thorne, S. H. Chemical Control of Protein Stability and Function in Living Mice. Nat. Med. 2008, 14 (10), 1123-1127.

(7) Su, L.; Li, A.; Li, H.; Chu, C.; Qiu, J. L. Direct Modulation of Protein Level in Arabidopsis. Mol. Plant 2013, 6 (5), 1711-1714.

(8) Zhang, J.; Yin, K.; Sun, J.; Gao, J.; Du, Q.; Li, H.; Qiu, J. L. Direct and Tunable Modulation of Protein Levels in Rice and Wheat with a Synthetic Small Molecule. Plant Biotechnol. J. 2018, 16 (2), 472-481.

(9) Armstrong, C. M.; Goldberg, D. E. An FKBP Destabilization Domain Modulates Protein Levels in Plasmodium falciparum. Nat. Methods 2007, 4 (12), 1007-1009.

(10) Brooks, C. F.; Johnsen, H.; van Dooren, G. G.; Muthalagi, M.; Lin, S. S.; Bohne, W.; Fischer, K.; Striepen, B. The Toxoplasma Apicoplast Phosphate Translocator Links Cytosolic and Apicoplast Metabolism and Is Essential for Parasite Survival. Cell Host Microbe 2010, 7 (1), 62-73.

(11) Madeira da Silva, L.; Owens, K. L.; Murta, S. M. F.; Beverley, S. M. Regulated Expression of the Leishmania Major Surface Virulence Factor Lipophosphoglycan Using Conditionally Destabilized Fusion Proteins. Proc. Natl. Acad. Sci. U. S. A. 2009, 106 (18), 7583-7588.

(12) Rakhit, R.; Edwards, S. R.; Iwamoto, M.; Wandless, T. J. Evaluation of FKBP and DHFR Based Destabilizing Domains in 
Saccharomyces Cerevisiae. Bioorg. Med. Chem. Lett. 2011, 21 (17), $4965-4968$.

(13) Jørgensen, F. P.; Madsen, D.; Meldal, M.; Olsen, J. V.; Petersen, M.; Granhøj, J.; Bols, M. Synthesis of Shld Derivatives, Their Binding the Destabilizing Domain and Influence on Protein Accumulation in Transgenic Plants. J. Med. Chem. 2019, 62 (10), 5191-5216.

(14) Dunyak, B. M.; Gestwicki, J. E. Peptidyl-Proline Isomerases (PPIases): Targets for Natural Products and Natural Product-Inspired Compounds. J. Med. Chem. 2016, 59 (21), 9622-9644.

(15) Wu, X.; Wang, L.; Han, Y.; Regan, N.; Li, P. K.; Villalona, M. A.; Hu, X.; Briesewitz, R.; Pei, D. Creating Diverse Target-Binding Surfaces on FKBP12: Synthesis and Evaluation of a Rapamycin Analogue Library. ACS Comb. Sci. 2011, 13 (5), 486-495.

(16) Guo, Z.; Hong, S. Y.; Wang, J.; Rehan, S.; Liu, W.; Peng, H.; Das, M.; Li, W.; Bhat, S.; Peiffer, B.; Ullman, B. R.; Tse, C. M.; Tarmakova, Z.; Schiene-Fischer, C.; Fischer, G.; Coe, I.; Paavilainen, V. O.; Sun, Z.; Liu, J. O. Rapamycin-Inspired Macrocycles with New Target Specificity. Nat. Chem. 2019, 11 (3), 254-263.

(17) Holt, D. A.; Luengo, J. I.; Yamashita, D. S.; Oh, H. J.; Konialian, A. L.; Yen, H. K.; Rozamus, L. W.; Brandt, M.; Bossard, M. J.; Levy, M. A.; Eggleston, D. S.; Liang, J.; Schultz, L. W.; Stout, T. J.; Clardy, J. Design, Synthesis, and Kinetic Evaluation of High-Affinity FKBP Ligands and the X-Ray Crystal Structures of Their Complexes with FKBP12. J. Am. Chem. Soc. 1993, 115 (22), 9925-9938.

(18) Keenan, T.; Yaeger, D. R.; Courage, N. L.; Rollins, C. T.; Pavone, M. E.; Rivera, V. M.; Yang, W.; Guo, T.; Amara, J. F.; Clackson, T.; Gilman, M.; Holt, D. A. Synthesis and Activity of Bivalent FKBP12 Ligands. Bioorg. Med. Chem. 1998, 6 (8), 13091335.

(19) Gaali, S.; Kirschner, A.; Cuboni, S.; Hartmann, J.; Kozany, C.; Balsevich, G.; Namendorf, C.; Fernandez-Vizarra, P.; Sippel, C.; Zannas, A. S.; Draenert, R.; Binder, E. B.; Almeida, O. F. X.; Rühter, G.; Uhr, M.; Schmidt, M. V.; Touma, C.; Bracher, A.; Hausch, F. Selective Inhibitors of the FK506-Binding Protein 51 by Induced Fit. Nat. Chem. Biol. 2015, 11 (1), 33-37.

(20) Jørgensen, F. P.; Bols, M. An Inexpensive and Scalable Synthesis of Shld. J. Org. Chem. 2018, 83 (11), 6050-6055.

(21) Meldal, M.; Christensen, S. F. Microparticle Matrix Encoding of Beads. Angew. Chem., Int. Ed. 2010, 49 (20), 3473-3476.

(22) Farrer, R. A.; Copeland, G. T.; Previte, M. J. R.; Okamoto, M. M.; Miller, S. J.; Fourkas, J. T. Production, Analysis, and Application of Spatially Resolved Shells in Solid-Phase Polymer Spheres. J. Am. Chem. Soc. 2002, 124 (9), 1994-2003.

(23) Braeckmans, K.; De Smedt, S. C.; Roelant, C.; Leblans, M.; Pauwels, R.; Demeester, J. Encoding Microcarriers by Spatial Selective Photobleaching. Nat. Mater. 2003, 2 (3), 169-174.

(24) Battersby, B. J.; Bryant, D.; Meutermans, W.; Matthews, D.; Smythe, M. L.; Trau, M. Toward Larger Chemical Libraries: Encoding with Fluorescent Colloids in Combinatorial Chemistry. J. Am. Chem. Soc. 2000, 122 (9), 2138-2139.

(25) Fenniri, H.; Ding, L.; Ribbe, A. E.; Zyrianov, Y. Barcoded Resins: A New Concept for Polymer-Supported Combinatorial Library Self-Deconvolution. J. Am. Chem. Soc. 2001, 123 (33), $8151-8152$

(26) Kassel, D. B. Combinatorial Chemistry and Mass Spectrometry in the 21 st Century Drug Discovery Laboratory. Chem. Rev. 2001, 101 (2), 255-267.

(27) Kolos, J. M.; Voll, A. M.; Bauder, M.; Hausch, F. FKBP Ligands-Where We Are and Where to Go? Front. Pharmacol. 2018, 9, $1-25$.

(28) Berthet, M.; Davanier, F.; Dujardin, G.; Martinez, J.; Parrot, I. MgI2-Mediated Chemoselective Cleavage of Protecting Groups: An Alternative to Conventional Deprotection Methodologies. Chem. Eur. J. 2015, 21 (31), 11014-11016.

(29) Auzanneau, F. -I; Meldal, M.; Bock, K. Synthesis, Characterization and Biocompatibility of PEGA Resins. J. Pept. Sci. 1995, 1 (1), $31-44$.
(30) Meldal, M. The One-Bead Two-Compound Assay for Solid Phase Screening of Combinatorial Libraries. Biopolymers 2002, 66 (2), 93-100.

(31) Noblin, D. J.; Page, C. M.; Tae, H. S.; Gareiss, P. C.; Schneekloth, J. S.; Crews, C. M. A HaloTag-Based Small Molecule Microarray Screening Methodology with Increased Sensitivity and Multiplex Capabilities. ACS Chem. Biol. 2012, 7 (12), 2055-2063.

(32) Hu, H.; Nikitin, S. V.; Berthelsen, A. B.; Diness, F.; Schoffelen, S.; Meldal, M. Sustainable Flow Synthesis of Encoded Beads for Combinatorial Chemistry and Chemical Biology. ACS Comb. Sci. 2018, 20 (8), 492-498.

(33) Castro, V.; Blanco-Canosa, J. B.; Rodriguez, H.; Albericio, F. Imidazole-1-Sulfonyl Azide-Based Diazo-Transfer Reaction for the Preparation of Azido Solid Supports for Solid-Phase Synthesis. ACS Comb. Sci. 2013, 15 (7), 331-334.

(34) Li, M.; Hoeck, C.; Schoffelen, S.; Gotfredsen, C. H.; Meldal, M. Specific Electrostatic Molecular Recognition in Water. Chem. - Eur. J. 2016, 22 (21), 7206-7214. 